\title{
State Effects of Two Forms of Meditation on Prefrontal EEG Asymmetry in Previously Depressed Individuals
}

\author{
Thorsten Barnhofer • Tobias Chittka • \\ Helen Nightingale $\cdot$ Claire Visser $\cdot$ Catherine Crane
}

Published online: 18 March 2010

(C) The Author(s) 2010. This article is published with open access at Springerlink.com

\begin{abstract}
We investigated state effects of two forms of meditation on electroencephalography prefrontal $\alpha$ asymmetry, a global indicator of approach versus withdrawal motivation and related affective state. A clinical series of previously depressed individuals were guided to practice either mindfulness breathing meditation $(N=8)$ or a form of meditation directly aimed at cultivating positive affect, loving kindness or metta meditation $(N=7)$. Prefrontal asymmetry was assessed directly before and after the 15-min meditation period. Results showed changes in asymmetry towards stronger relative left prefrontal activation, i.e., stronger approach tendencies, regardless of condition. Further explorations of these findings suggested that responses were moderated by participants' tendencies to engage in ruminative brooding. Individuals high in brooding tended to respond to breathing meditation but not loving kindness meditation, while those low in brooding showed the opposite pattern. Comparisons with an additionally recruited "rest" group provided evidence suggesting that changes seen were not simply attributable to habituation. The results indicate that both forms of meditation practice can have beneficial state effects on prefrontal $\alpha$-asymmetry and point towards differential indications for offering them in the treatment of previously depressed patients.
\end{abstract}

T. Barnhofer $(\bowtie) \cdot H$. Nightingale $\cdot$ C. Visser $\cdot$ C. Crane Department of Psychiatry, Warneford Hospital, Oxford University, Oxford OX3 7JX, UK

e-mail: thorsten.barnhofer@psych.ox.ac.uk

T. Chittka

University of Dresden,

Dresden, Germany
Keywords Meditation · Mindfulness · Loving kindness · Depression $\cdot$ Prefrontal $\alpha$-asymmetry

\section{Introduction}

Over the recent years, there has been an increasing interest in using mindfulness meditation as a form of mental training in the treatment of emotional disorders. Mindfulness-Based Cognitive Therapy (MBCT; Segal et al. 2002) has been found to be effective in reducing risk for relapse to depression, and preliminary evidence suggests beneficial effects of mindfulness-based approaches in a broad spectrum of other disorders including anxiety disorders, alcohol abuse, and eating disorders (for an overview, see Didonna 2009). The general rationale guiding the use of mindfulness meditation in these contexts is the idea that through meditation practice patients will learn to establish a mode of mind that helps to preempt engagement in maladaptive habitual patterns of processing and that is conducive to the cultivation of positive affect. Indeed, much interest in the use of mindfulness meditation has been raised by findings suggesting that it may have lasting effects on psychological functioning and brain activity. In a seminal study, Davidson et al. (2003) found that intensive training in mindfulness meditation produced significant changes in prefrontal $\alpha$ asymmetry, a measure of relative right versus left prefrontal activation that has been shown to predict individual tendencies towards approach versus avoidance and related affective states.

In the current study, we were interested in investigating state effects of meditation. MBCT utilizes brief and portable meditation practices as a means of generalizing the cultivation of mindfulness to a wider range of situations in daily life, but also, particularly in later stages of the program, as a way of 
skillfully responding to negative mood. The assumption guiding this latter use is that, in situations where mood is low, brief mindfulness meditation may help participants to become more aware of their current thoughts and feelings, enabling them to respond more adaptively. Additionally, brief mindfulness meditation, through its decentering function, may interrupt the downward cycles of negative mood and cognition which might otherwise persist and escalate (Lau et al. 2004) and hence have positive effects on subsequent affective state. However, while potentially conducive to the cultivation of positive affect, during mindfulness meditation, participants are simply encouraged to observe their current experience in an open and nonjudgmental way. Cultivation of positive affect, thus, remains an implicit or indirect intention of mindfulness practice.

In contrast to these implicit aspects of some mindfulness practices, the practice of loving kindness is explicitly aimed at cultivating positive or "wholesome" states of mind (see Salzberg 1995). While not part of the manualized approaches of MBCT and MBSR, many mindfulness teachers complement their teaching of mindfulness with loving kindness meditation. The term loving kindness or metta, in the Pali language, refers to unconditional regard and nonexclusive love for all beings and is one of the four main Buddhist virtues. In metta meditation, the practitioner silently repeats a set of phrases, cultivating a felt sense of loving kindness, first for themselves and then, in several steps, to other beings and, ultimately, all beings everywhere. A recent study found a significant increase in positive emotions and personal resources as well as overall life satisfaction in a group of previously naïve subjects who practiced this form of meditation for half an hour daily over a period of several weeks (Fredrickson et al. 2008). Given such effects, the practice of loving kindness may seem to be a helpful addition to MBCT. However, clinical impressions suggest that participants' responses to this practice differ widely and that a considerable number of patients seem to find it difficult to engage with. We wanted to investigate why this might be and whether any such difficulty could be shown on an objective measure.

To this end, we investigated state effects of mindfulness of breathing and loving kindness meditations on electroencephalography (EEG) prefrontal $\alpha$-asymmetry. Prefrontal asymmetry is a global indicator of affective style that is computed as the difference in activation, inversely indexed by EEG $\alpha$-power density, in right and left prefrontal cortex regions. A large body of research on this parameter has shown relative left prefrontal activation to be related to stronger tendencies towards approach motivation and positive affect and relative right prefrontal activation to be related to stronger tendencies towards withdrawal motivation and negative affect (Davidson 2004). As described above, previous research has found that intensive practice of mindfulness meditation can change resting levels of prefrontal $\alpha$-asymmetry, changes that have been assumed to reflect trait-level alterations in relative approach/withdrawal motivation and affect (Davidson et al. 2003). In contrast to this, the current study was interested in investigating state effects. Psychometric studies have found that resting state assessments of EEG prefrontal asymmetry reflect both state and trait components with trait components estimated to account for about $40 \%$ to $60 \%$ of the variance (Hagemann et al. 2005), suggesting that the parameter may be sufficiently sensitive for the assessment of staterelated changes. We hypothesized that previously depressed participants practicing loving kindness meditation, explicitly aimed at cultivating positive affect, would show significantly stronger changes towards relative left prefrontal activation, i.e., approach motivation and related positive affect, than participants practicing breathing meditation, assumed to have only indirect effects on affective state.

Following clinical observation that individuals may differ widely in their response to loving kindness meditation, we explored the role of habitual tendencies in how individuals reflect on their experiences as a potential factor involved in determining initial response. Here, we were particularly interested in tendencies towards brooding. Brooding, a form of "moody pondering" assessed through participants' reported tendency to self-chastise at times of low mood, with thoughts such as "why can't I handle things better" or "why do I always react this way", has been found to be an important factor in the maintenance of negative mood states (Treynor et al. 2003). Its aim of reducing discrepancies between current and desired mood states through self-critical thinking seems to run counter to the idea of unconditional regard that is cultivated in loving kindness meditation. With this in mind, we expected that individuals who habitually engaged in this type of thinking might find it more difficult to benefit from metta meditation practice, at first exposure.

Finally, since both forms of meditation practice involve a period of sitting at rest, albeit engaged in different mental activities, in order to increase interpretability of our findings, we added, at a later date, a group of participants who simply rested between EEG assessments instead of meditating. This group provides an estimate of changes to be expected in resting prefrontal asymmetry due to unspecific factors such as habituation to the laboratory environment and EEG recording equipment.

\section{Method}

Participants

Participants were recruited through posters and advertisements in local publications and websites. Individuals 
interested in taking part made contact via telephone or email and received further information on the experiment as well as inclusion and exclusion criteria. Those who felt that they would qualify for the study were invited for a structured clinical interview (Mini International Neuropsychiatric Interview (MINI), Sheehan et al. 1998) conducted by a trained psychologist. In order to be included in the study, participants had to have a history of at least one previous episode of major depression and to be in recovery for at least 8 weeks as defined by the presence of no more than one of the symptoms of depression during a given week. Sixteen individuals took part in the initial interview, one of whom did not return for the second session in which the actual experiment was conducted. The final sample, therefore, consisted of 15 participants, eight of whom were randomized to guided breathing meditation and seven of whom were randomized to guided loving kindness meditation. The two groups were comparable in terms of gender distribution, current depressive symptoms (Beck Depression Inventory (BDI)), ruminative tendencies in general and tendencies to brood in particular (Response Style Questionnaire (RSQ) total and RSQ brooding subscale), age of onset of the depressive disorder, and number of previous episodes, all $p>0.20$. Mean age in the breathing meditation group was higher, although not significantly, $p=0.48$, than in the loving kindness meditation group due to one participant aged 63 who in all other regards showed data comparable to younger participants. Means and standard deviations of these characteristics are listed in Table 1.

\section{Procedure}

During the initial assessment, participants were interviewed with the MINI and then filled in several questionnaires. The experimental session took place 1 week after the initial assessment. Upon arrival in the lab, participants were greeted and given a short tour of the lab. After having given their informed consent, they were seated in the EEG room where they completed further questionnaires before the EEG recording was prepared and electrodes attached. Resting EEG was assessed over 2 min before and after the guided meditation while participants sat in the dimly lit room. For the time of the EEG recording, participants were instructed to keep their eyes shut and to simply rest. The guided meditations were presented via speakers, and participants were asked to follow the recorded instructions as best they could and to try and remain open-minded even if the meditation seemed unusual to them.

\section{Materials}

Guided Meditations Recordings of the guided meditations were both $15 \mathrm{~min}$ in length and were comparable in terms of the ratio of verbal instructions to silent periods in which participants kept practicing for themselves.

For the guided breathing meditation, participants were instructed to focus their attention on the sensations of the breath wherever in their body it seemed most vivid to them, to keep the attention on the breath, and, when their mind had wandered, to notice the content of the distraction and then to refocus their attention on the breath without blaming themselves for having lost their focus.

In the metta meditation, participants were instructed to start by thinking about a good quality that they had or a good thing that they had done or, if nothing came to mind, to just think of their own wish to be happy. They were then asked to direct loving kindness towards themselves by silently repeating four phrases: "May I be safe and protected. May I be happy and contented. May I be healthy and whole. May I live with ease." Once they had established loving kindness for themselves, they were asked to use the phrases to cultivate loving kindness towards others, first to someone close, then someone neutral, then someone they did not know first-hand, and finally to all people (see Salzberg 1995).

Questionnaires Severity of current symptoms of depression was assessed using the BDI-II (Beck et al. 1996). The BDI-
Table 1 Participants' characteristics

BDI-II Beck Depression Inventory II, $R S Q$ Response Style Questionnaire Rumination Scale

\begin{tabular}{lcc}
\hline & Breathing & Loving kindness \\
\hline$N$ (female/male) & $8(7 / 1)$ & $7(5 / 2)$ \\
Age $M(\mathrm{SD})$ & $31.6(14.3)$ & $24.4(3.8)$ \\
BDI-II $M(\mathrm{SD})$ & $4.5(5.9)$ & $2.1(3.7)$ \\
Age at onset $M(\mathrm{SD})$ & $20.1(6.5)$ & $17.9(5.1)$ \\
Previous episodes Mdn (range) & $2.0(1-16)$ & $2.5(1-10)$ \\
RSQ total $M(\mathrm{SD})$ & $51.3(12.2)$ & $55.8(15.9)$ \\
RSQ brooding $M(\mathrm{SD})$ & $11.8(3.7)$ & $12.2(4.6)$ \\
RSQ depressive rumination $M(\mathrm{SD})$ & $27.7(6.2)$ & $30.4(9.7)$ \\
RSQ reflection $M(\mathrm{SD})$ & $11.7(4.3)$ & $13.1(3.6)$ \\
\hline
\end{tabular}


II is a well-established self-report instrument containing 21 items that assess the presence and severity of different depressive symptoms over the last 2 weeks.

In order to assess tendencies towards brooding, we used the Ruminative Responses Scale (RRS), a self-report measure of rumination, designed by Nolen-Hoeksema. Recent research using this questionnaire has differentiated between three components of rumination, rumination about symptoms of depression, reflective pondering, and brooding, indicating that it is the latter component that predicts increases in depressive symptoms as an outcome (see Treynor et al. 2003). The brooding subscale of the RRS consists of five items. The internal consistency of this subscale in the current study was Cronbach's $\alpha=0.82$.

\section{EEG Recording and Quantification}

EEG was recorded from sites Fp1, Fp2, AF3, AF4, Fz, F3, F4, F7, F8, Fc1, Fc2, Fc5, Fc6, Cz, C3, C4, T7, T8, Cp1, Cp2, Cp5, Cp6, Tp9, Tp10, Pz, P3, P4, P7, P8, O1, O2, A1, and $\mathrm{A} 2$ using an Easy Cap system with sintered $\mathrm{Ag} / \mathrm{AgCl}$ electrodes. All sites were referenced to the average during recording and re-referenced off-line to derive an averaged ears $[(\mathrm{A} 1+\mathrm{A} 2) / 2]$ reference, which was used as the reference of choice for all analyses. EOG was recorded from the epicanthus of each eye and from supraorbital and infraorbital positions.

Impedances were reduced to less than $5 \mathrm{k} \Omega$, and differences between homologous sites were less than $2 \mathrm{k} \Omega$ in all cases. Signals were amplified with a BrainVision Quickamp with 22-bit $\mathrm{A} / \mathrm{D}$ conversion and a resolution of $71.5 \mathrm{nV}$ (range $\pm 150 \mathrm{mV}$ ) and digitized at a rate of $500 \mathrm{~Hz}$. The recorded data were resampled to $512 \mathrm{~Hz}$, using spline interpolation, and filtered with a time constant of 03.s and an upper cutoff frequency of $40 \mathrm{~Hz}(-24 \mathrm{db} / \mathrm{Oct})$. The two 2-min recordings were divided into $1-\mathrm{s}$ epochs that overlapped by $50 \%$, resulting in 239 epochs for each of the recordings. To control for ocular artifacts, data were subjected to an algorithm that automatically rejected epochs for all channels when ocular signals exceeded $\pm 100 \mu \mathrm{V}$.

Artifact-free epochs were extracted through a Hamming window that tapered data at the distal $10 \%$ of each epoch and subjected to a fast Fourier transform to derive measures of spectral power density $\left(\mu \mathrm{V}^{2} / \mathrm{Hz}\right)$. The resulting spectra were averaged over all artifact-free episodes of the given recording, and power density within the $\alpha$-band $(8-13 \mathrm{~Hz})$ was extracted. Power density values were log-transformed using the natural $\log$ to normalize data. Frontal EEG asymmetry at midfrontal sites F4 and F3 was computed by subtracting left hemispheric power density from right hemispheric power density $[\ln (\alpha$ power density F4) $-\ln (\alpha$ power density F3)]. As $\alpha$ power density is inversely related to cortical activity, higher scores in this metric indicate relatively higher left-sided activity, i.e., stronger approach motivation.

\section{Results}

Changes in frontal EEG asymmetry from premeditation to postmeditation were analyzed using a repeated-measures analysis of variance (ANOVA) with time (pre versus post) as within-subjects and group (breathing meditation versus loving kindness meditation) as between-subjects factor. There was a significant main effect of time, $F(1,13)=$ $5.06, p=0.04$, reflecting a general increase in asymmetry scores from pre to post testing, i.e., an increase in relative left prefrontal activation indicating increased approach and positive affect. The time $\times$ group interaction failed to reach significance, $p=0.60$, indicating that the expected differential effect of meditation type, i.e., stronger increases in asymmetry following loving kindness meditation, was not present (breathing meditation: $M_{I-J}=-0.075, \mathrm{SE}=0.037$, $p=0.06$; loving kindness meditation: $M_{I-J}=-0.046$, $\mathrm{SE}=0.039, p=0.25$ ). Means and standard deviations of prefrontal asymmetry scores before and after meditation for both groups are depicted in Table 2.

In order to further investigate these findings, we explored asymmetry changes in participants who had described themselves as high or low in their tendency for brooding, as determined by median split. Visual inspection of mean and individual scores yielded a pattern suggesting that responders to loving kindness meditation tended to be low in brooding while responders to breathing meditation tend to be high in brooding (see Fig. 1a, b). In order to more formally test this interaction, we conducted a repeated-measures ANOVA with time (pre versus post) as within-subjects and meditation group (breathing versus loving kindness) and brooding (high versus low) as between-subjects factors which, consistent with the explorative findings, yielded a significant three-way time $\times$ meditation group $\times$ brooding interaction, $F(1,11)=4.86$, $p=0.05$.

Table 2 Means and standard deviations of prefrontal asymmetry scores before and after meditation in participants allocated to breathing $(N=8)$, loving kindness meditation $(N=7)$, or rest $(N=8)$

\begin{tabular}{|c|c|c|c|c|}
\hline & \multicolumn{2}{|c|}{ Before } & \multicolumn{2}{|l|}{ After } \\
\hline & $M$ & SD & $M$ & $\mathrm{SD}$ \\
\hline Breathing & 0.053 & 0.077 & 0.128 & 0.159 \\
\hline Loving kindness & 0.071 & 0.079 & 0.118 & 0.109 \\
\hline Rest & 0.073 & 0.104 & 0.048 & 0.088 \\
\hline
\end{tabular}



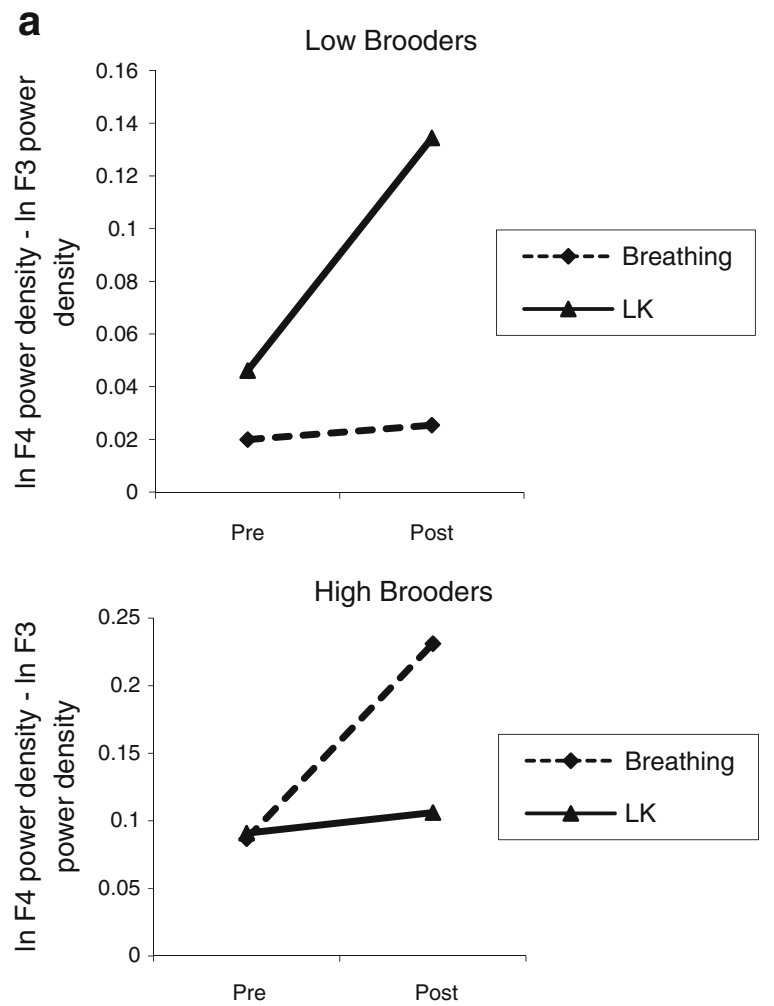

b

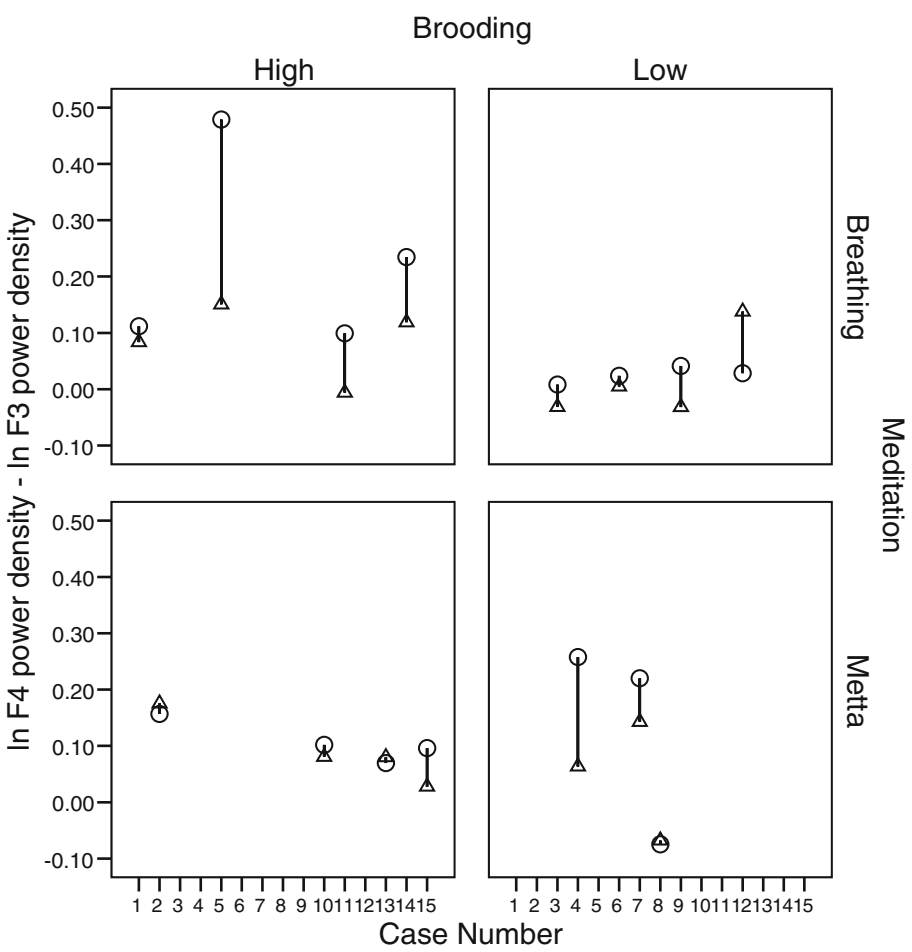

Fig. 1 Mean (a) and individual (b) prefrontal asymmetry scores at pre-meditation and post-meditation in participants allocated to breathing or loving kindness meditation split by level of brooding (high versus low).

However, separate time $\times$ meditation group repeatedmeasures ANOVAs, following up the three-way interaction, in the high- and low-brooding groups did not yield significant results. In the high-brooding group, the time $\times$ meditation group interaction reached trend levels, $F(1,6)=$ 3.69, $p=0.10$; follow-up tests with Bonferroni correction showed significant pre to post changes in the breathing meditation group, $M_{I-J}=-0.14, \mathrm{SE}=0.04, p=0.02$, while there was no significant change in the loving kindness meditation group, $M_{I-J}=-0.01, \mathrm{SE}=0.04, p=0.76$. In the low-brooding group, the time by meditation group interaction was nonsignificant, $p=0.27$; as were the follow-up tests: $M_{I-J}=-0.08, \mathrm{SE}=0.05, p=0.14$, for the loving kindness meditation, compared to $M_{I-J}=-0.01, \mathrm{SE}=0.04$, $p=0.90$, for the breathing meditation group.

\section{Comparison with Changes in a Rest Condition}

While the above findings suggest that the two meditations can bring about comparable changes in prefrontal asymmetry, it remained unclear from the current design whether these changes are actually due to the effects of the meditations or to unspecific factors such as habituation to the experimental situation over time. In order to get an estimate of the effect of unspecific factors, we recruited an additional sample of participants who took part in all aspects of the study as previously described but, instead of engaging in a brief meditation, were asked to simply rest and wait for $15 \mathrm{~min}$ between the two EEG assessments of the experimental part of the study. The group consisted of eight previously depressed people (four females, four males) with an average age of $M=36.6$ years, SD = 10.92. The mean BDI score at the time of the study was $M=6.00, \mathrm{SD}=7.16$. Comparison of pre and post scores in this group showed that asymmetry scores at $\mathrm{F} 4 / \mathrm{F} 3$ remained virtually unchanged (pre: $M=0.07, \mathrm{SD}=0.10$; post: $M=0.04, \mathrm{SD}=0.08$ ). A repeated-measures ANOVA comparing changes in this rest group against changes in the two meditation groups yielded a significant time $\times$ group interaction, $F(1,20)=4.55, p=0.04$.

\section{Discussion}

Contrary to our hypotheses, previously depressed participants who engaged in loving kindness meditation, a direct way of cultivating positive affect, did not show stronger changes in prefrontal asymmetry than previously depressed participants who engaged in breathing meditation, a form of meditation that is not explicitly aimed at producing positive affect. Instead, we found that in our sample both forms of meditation produced equally positive effects. Further exploratory analy- 
ses pointed towards a potential role of ruminative brooding in whether or not participants responded to the given meditation.

The finding that, even in previously depressed people naïve to meditation, brief guided practice can bring about significant positive changes in brain processes involved in affective state replicates results from another study that investigated state effects of mindfulness meditation in a sample of normal volunteers (Chan et al. 2008). These results support the use of brief meditation exercises in MBCT and suggest that they may serve as a helpful means of generalizing adaptive affective states to a wider range of situations in daily life. Prefrontal asymmetry has been found to reliably predict responses to emotion elicitors such as positive or negative film clips, pictures, and words (for an overview, see Coan and Allen 2004). In previously depressed patients, increased leftsided activation may have an important protective effect given that triggering of cognitive vulnerability processes is significantly facilitated in states of negative mood.

What can we make of the finding from the exploratory analyses showing that not all participants responded with positive changes to the meditations? We found that whether or not participants responded to a particular form seemed to be systematically related to ruminative brooding. Most of those who were high in brooding did not seem to respond to metta, while most of those who were low did and vice versa for breathing meditation. Although based on very small numbers, tentative interpretation of these findings may provide some help in understanding potential difficulties previously depressed patients may encounter when first engaging with these practices.

Brooding arises as the results of continuing attempts to reduce discrepancies between current and desired states or aspects of the self and, thus, runs counter to the idea of unconditional regard that is cultivated in loving kindness mediation. Because of this, individuals with strong tendencies towards brooding may find the practice of loving kindness difficult to relate to. Breathing meditation, on the other hand, is likely to divert attention away from selfdiscrepancies and may thus not only be more accessible to those high in brooding but might also have positive effects by effectively reducing rumination through distraction and reducing negative mood, at least in the short term (NolenHoeksema and Morrow 1990). Similar differential responses have been reported by Salmon et al. (1998), who observed that pain patients who experience anxiety more somatically prefer more "cognitive" forms of meditation (e.g., sitting), while those who experience anxiety mainly as a cognitive phenomenon prefer more active forms of meditation such as mindful walking or yoga.

Interpretation of these findings needs to take into account several limitations. First, because the initial design of the study was restricted to the comparison of the two forms of meditation, it was difficult to rule out the possibility that positive changes occurred simply due to unspecific factors such as habituation. Testing this alternative explanation was possible only through adding a further sample of previously depressed people post hoc. Because of this, participants could not be distributed randomly to the different conditions, leading to slight differences between this control group and the two initial groups, i.e., in gender distribution. While this leaves some minor concerns with regard to comparability of the groups, these data provide some reassurance that the effects seen in the meditation conditions were unlikely to be due to habituation. The lack of change in the control group is also in line with previous studies that have found prefrontal asymmetry scores to show high stability at least over shorter periods of time (Tomarken et al. 1992). Second, because of the small sample size of the study, it is possible that the fact that we did not find a difference between loving kindness and breathing meditation is simply due to a lack in power to detect such differential effects. However, seeing that the meditations produced virtually identical effects it appears unlikely that even with higher numbers of participants significant differences would have emerged. Third, the participants in our study were all naïve to meditation and findings and thus only reflect initial responses to these techniques which may change with repeated practice.

In conclusion, the current findings suggest that brief meditation techniques can bring about significant changes in brain activity underlying affective state. However, they also suggest that participants can differ widely in their initial response to particular forms of meditation. These findings therefore underscore the importance of introducing meditation techniques in a way that encourages participants to reflect compassionately on their initial responses to the practices, particularly if difficult, and which emphasizes that initial experiences may not be predictive of potential long-term benefits.

Acknowledgments This research was supported by a grant from the Wellcome Trust and Nuffield Bursaries to Helen Nightingale and Clare Visser. The authors would like to thank Prof J. Mark G. Williams for helpful comments on this manuscript and for his support in the conducting of this research.

Open Access This article is distributed under the terms of the Creative Commons Attribution Noncommercial License which permits any noncommercial use, distribution, and reproduction in any medium, provided the original author(s) and source are credited.

\section{References}

Beck, A. T., Steer, R. A., \& Brown, G. K. (1996). Manual for the BDI-II. San Antonio: Psychological Corporation.

Chan, A. S., Han, Y. M. Y., \& Cheung, M.-C. (2008). Electroencephalographic measurements of mindfulness-based triarchic body- 
pathway relaxation technique: a pilot study. Applied Psychophysiology and Biofeedback, 33, 39-47.

Coan, J. A., \& Allen, J. J. B. (2004). Frontal EEG asymmetry as a moderator and mediator of emotion. Biological Psychology, 67, $7-49$.

Davidson, R. J. (2004). What does the prefrontal cortex "do" in affect: Perspectives on frontal EEG asymmetry research. Biological Psychology, 67, 219-233.

Davidson, R. J., Kabat-Zinn, J., Schumacher, J., Rosenkranz, M., Muller, D., Santorelli, S. F., et al. (2003). Alterations in brain and immune function produced by mindfulness meditation. Psychosomatic Medicine, 65, 564-570.

Didonna, F. (Ed.). (2009). Clinical handbook of mindfulness. New York: Springer.

Fredrickson, B. L., Cohen, M. A., Coffey, K. A., Pek, J., \& Finkel, S. M. (2008). Open hearts build lives: Positive emotions, induced through loving-kindness meditation, build consequential personal resources. Journal of Personality and Social Psychology, 95, 1045-1062.

Hagemann, D., Hewig, J., Seifert, J., Naumann, E., \& Bartussek, D. (2005). The latent state-trait structure of resting EEG asymmetry: Replication and extension. Psychophysiology, 42, 740-752.

Lau, M. A., Segal, Z. V., \& Williams, J. M. G. (2004). Teasdale's differential activation hypothesis: implications for mechanisms of depressive relapse and suicidal behaviour. Behaviour Research and Therapy, 42, 1001-1017.
Nolen-Hoeksema, S., \& Morrow, J. (1990). Effects of responses to depression on the remediation of depressive affect. Journal of Personality \& Social Psychology, 58, 519-527.

Salmon, P. G., Santorelli, S. F., \& bKabat-Zinn, J. (1998). Intervention elements promoting adherence to mindfulness-based stress reduction programs in the clinical behavioral medicine setting. In S. A. Shumaker, E. B. Schron, J. K. Ockene, \& W. L. McBee (Eds.), The handbook of health behavior change (pp. 239-266). New York: Springer.

Salzberg, S. (1995). Loving-kindness: The revolutionary art of happiness. Boston: Shambala.

Segal, Z. V., Williams, J. M. G., \& Teasdale, J. D. (2002). Mindfulnessbased cognitive therapy for depression: a new approach to preventing relapse. New York: Guilford.

Sheehan, D. V., Lecrubier, Y., Sheehan, K., Harnett, A. P., Janava, J., Weiller, E., et al. (1998). The Mini-International Neuropsychiatric Interview (M.I.N.I): The development and validation of a structured psychiatric interview for DSM-IV and ICD-10. Journal of Clinical Psychology, 59, 22-33.

Tomarken, A. J., Davidson, R. J., Wheeler, R. E., \& Kinney, L. (1992). Psychometric properties of resting anterior EEG asymmetry: Temporal stability and internal consistency. Psychophysiology, 29, 576-592.

Treynor, W., Gonzalez, R., \& Nolen-Hoeksema, S. (2003). Rumination reconsidered: A psychometric analysis. Cognitive Therapy and Research, 27, 247-259. 